\title{
Special section: crystallography and properties of metal organic framework (MOF) compounds
}

Research on metal-organic framework (MOF) materials has been a fast-growing field over the past decade. The interest and excitement in these materials are evidenced by the explosion in the number of highly cited MOF-related papers published in recent years. The increasing attention is partly owing to the wide potential applications, including gas sorption, gas storage, gas separation, catalysis, sensors, drug delivery, imaging, electronic devices, and environmental sustainability. The development and fundamental understanding of the properties of MOF materials are therefore important to technological needs, and they are at the forefront of materials research.

Crystallography provides the foundation of structure property relationships. It connects the fundamental understanding of materials with their applications. As functional MOF materials are envisioned to play an important role in our modern society and future economic and social success, the underlying crystallographic information for these materials is of utmost importance for many research communities.

While there have been a large number of papers dealing with structures of MOFs in many high-ranking journals, there has not been focused effort that highlights the current state-of-the-art capabilities developed for university laboratory use or available at large-scale user facilities that allow materials to be probed in-situ, in-operando, or multiplexed with other analytical techniques. Therefore, Powder Diffraction will be publishing special journal sections that will provide an important venue for authors and readers to recognize and appreciate the impact of these capabilities in understanding the structural implications on the function of MOFs.

The goal of the special sections is to focus on the latest developments in the cross-disciplinary fields of structure science and MOF materials. The papers will be published in the special sections throughout the four issues of the 2019 Powder Diffraction. Progress in measurement capabilities with in situ, in operando, multi-modal, and high-pressure crystallographic techniques is important areas for these journal sections to address. The types of materials of interest include bulk powders, single crystals, amorphous solids, thin films, and nanostructures. The special sections will encompass up-to-date research topics of MOFs as well as with reviews that present new evaluation and analyses of published work.
The content of the manuscripts will include structure/property relationships (properties of interest are catalytic, sorption, mechanical, gas storage, separations, energy harvesting, luminescence, energy conversion, electrical, and magnetic properties), instrumentation/techniques (and combined use) for analysis of MOFs, software tools for the analysis of MOFs, guest-host interactions, materials for industrial applications, crystallography and computational modeling, new crystallographic and powder diffraction data, and in situ, in operando, and non-ambient crystallography.

In this particular issue of Powder Diffraction, we have one review and one technical paper. Our review paper (XRDDSC: a screening tool for identifying effective MOFs for selective gas sorption from humid gas streams) is an invited paper by Professor John Parise concerning the combined use of XRD and DSC to characterize gas sorption processes. The second paper is by Professor Lan Li's group concerning using modeling techniques to understand the $\mathrm{CO}_{2}$ adsorption mechanism in a porous octahedral molecular sieve system. The guest editors anticipate that these papers will serve the community as the reference for modern measurement capabilities, data analysis, and materials insight.

\section{Guest Editors:}

Dr. Craig Brown is currently a staff chemist at the National Institute of Standards and Technology (NIST) Center for Neutron Research (NCNR) where he leads the Structure and Dynamics of Materials Team that supports a suite of neutron instruments. He is also an Affiliated Assistant Professor in the Chemical Engineering Department at the University of Delaware.

Dr. Winnie Wong-Ng is currently a research chemist (co-project leader) in the Materials for Energy and Sustainable Development Group of the Materials Measurement Science Division of NIST. She is also an adjunct professor at the Materials Science and Engineering Department of Boise State University.

Craig Brown Winnie Wong-Ng Guest Editors 